\title{
The effect of meditation on shooting performance
}

\author{
Erik E Solberg, Kurt-Arne Berglund, Øyvind Engen, Øivind Ekeberg, Mitch Loeb
}

\begin{abstract}
Objective-To study effects of meditation on the shooting performance.

Methods-25 elite shooters were investigated in an independent groups design. The results in standardised test shootings indoors and in ordinary competitions outdoors were assessed before and after regular meditation training for the experimental group. The experience of tension during the test shootings was self recorded on a visual analogue scale (VAS).

Results-The competition results in the outdoor season (1993), just after the meditation training period, compared with the results the previous season (1992), were better in the meditation group $(P<0.05)$. No significant difference between the groups was observed in the test shootings before and after the relaxation intervention. A significant association was shown between low tension and the results in the test shootings (correlation $r=0.42, P<$ 0.0001 ; Wilcoxon rank sum test, $z=-3.36$, $P<0.001) ; 18 \%\left(=r^{2}\right)$ of the variance in performance was explained by tension.

Conclusions-Meditation may enhance competitive shooting performance.

(Br $\mathcal{F}$ Sports Med 1996;30:342-346)
\end{abstract}

Key terms: shooting competition; meditation; mental training; relaxation techniques

The use of psychological interventions in sports has become increasingly popular ${ }^{1}$ and has been described in several publications. ${ }^{1-8}$ The efficacy of such interventions has been poorly documented. Recent reviews, however, suggest that more than $85 \%$ of studies addressing the issue have reported significant improvement of performance after mental training. ${ }^{1-3}$ On the other hand, the validity of these studies has been questioned. Causality between the psychological intervention and performance could be inferred in fewer of the studies. The lack of manipulation checks, appropriate controls, and clearly described interventions has been pointed out as other major research problems. ${ }^{12}$

A variety of psychological interventions has been used, and meditation-not the most frequently used technique-may be classified as relaxation based. ${ }^{1}$ The technique is normally used in sports for the purpose of upgrading the rate of recovery or enhancing performance by improving the handling of anxiety pressure. Its possible effect on sports performance, however, has not been well investigated. Among non-athletes, different types of meditation are widespread. Some effects related to relaxation and anxiety have been described ${ }^{10}$ which can explain in part the beneficial effects hypothesised in sports.

Relevant relaxation and arousal indices such as blood lactate, heart rate, and oxygen uptake are all considerably lowered after meditation. ${ }^{10-13}$ Meditation may relieve tension and anxiety..$^{10}$ These findings provide evidence that a relaxational effect of meditation is taking place. There is a possible link between an increased rate of recovery, a better handling of anxiety and tension, and enhanced performance.

Shooting is a type of sport assumed to be sensitive to tension and anxiety pressure. The level of shooting performance can be precisely evaluated by reading the exact score at the target, a benefit for research purposes. Given that research indicates that relaxation based interventions are some of the most effective, ${ }^{13}$ we sought to address the issue of meditation and its effect on shooting performance.

The primary aim of the study was to explore whether meditation would enhance elite shooters' performance in test shootings and competitions. A possible association between their tension and the level of performance was assessed as a secondary aim.

\section{Methods}

SUBJECTS

Elite shooters were openly invited from-for practical reasons-five shooting clubs deliberately chosen from the Oslo area. Being an inclusion criterion, all the shooters had performed above 236 (of a maximum 250) points in a standard programme of the National Rifle Association of Norway (NRAN): five shots prone $(1.5 \mathrm{~min})$, five shots standing $(3 \mathrm{~min})$, five shots kneeling ( $2 \mathrm{~min}$ ), and 10 consecutive shots (three standing, three kneeling, and four prone) in $3 \mathrm{~min}$, in total 25 shots giving a maximum score of 250 points.

Twenty five shooters of both sexes (21 males and four females) responded. They had a median age of 25 (range 18 to 46 ) years. Before the relaxation intervention no significant differences were detected between the groups in either the mean competition score (1992) or the results of the mean baseline test shootings (figs 1 and 2). The keen shooter takes part in 25-40 competitions per season on average (NRAN data). NRAN files showed that in the outdoor season after the intervention (1993), the study subjects had participated in a median of 27 competitions (range 5-64).

\section{PROCEDURES}

Independent variable

ACEM meditation ${ }^{14}{ }^{15}$ was used as the meditation technique, a natural choice as it is the most 


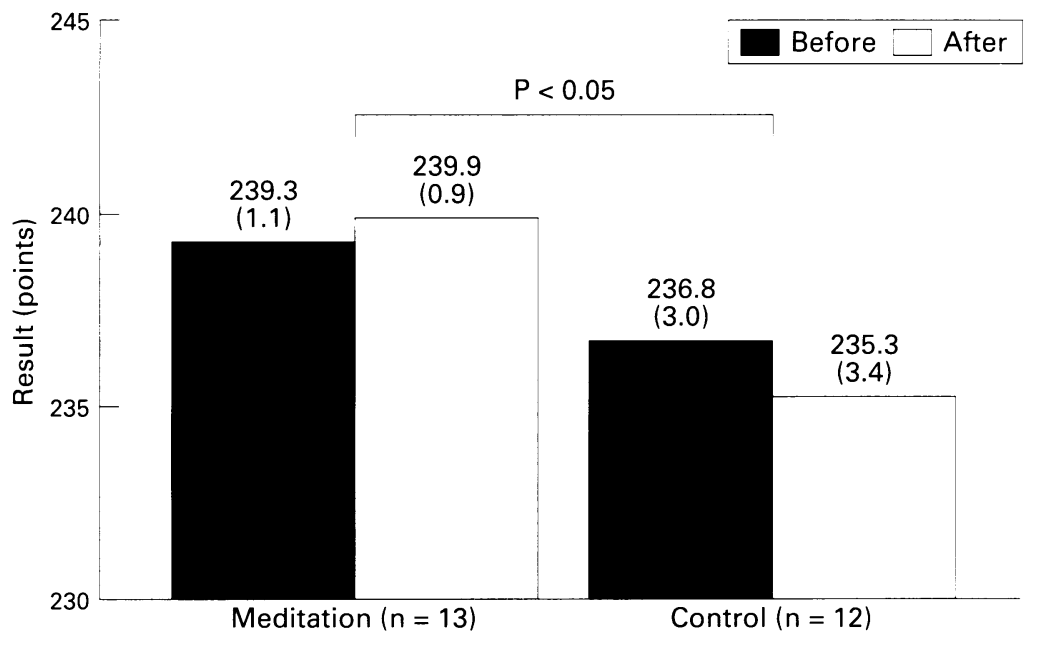

Figure 1 The mean results (with SEM values) of the three best competitions in the outdoor season before the intervention (1992) and after it (1993).

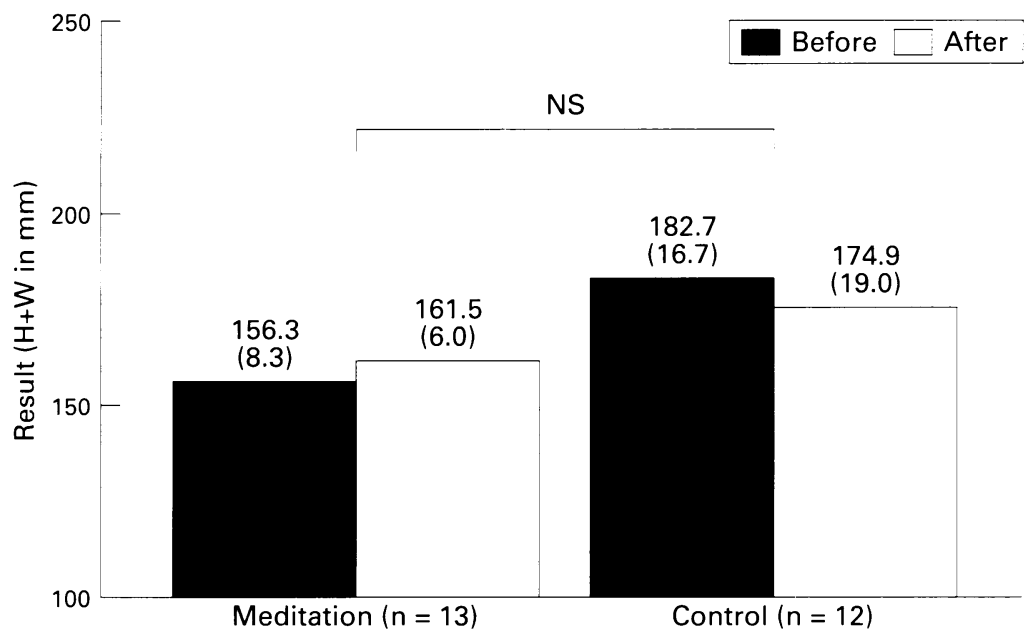

Figure 2 The mean test shooting results (as height + width in mm, with SEM values) of 50 shots from each of the 25 participants before and after the intervention. Higher score shows lower performance.
RELAXATION INTERVENTION

After an initial test shooting, the participants were randomly assigned to one group $(n=13)$ learning ACEM meditation once a week for seven weeks (additional details available from ACEM International homepage, http:// www.sn.no/home/acem/acem.htlm), or a control group $(\mathrm{n}=12)$ not receiving any kind of mental training (see flow chart in fig 3). The shooters did not receive any particular instructions to use meditation before the shooting bout.

The relaxation technique involves a nondirected repetition of a simple sound, and was practised 30 minutes daily at home during the intervention period. The practice (which has been precisely described ${ }^{1415}$ ) is fairly similar to transcendental meditation, ${ }^{11}$ but has a different background and is explained in western, non-cultic, psychophysiological terms. Methodologically it can be classified as a kind of mental training stimulating the relaxation responses through free floating awareness, as opposed to most techniques used in sports that are based on concentration.

COMPETITION SHOOTING

The NRAN standard programme, which has been established in Scandinavia for several years, was used to assess the competition shooting. The classification is based on the mean of the three best results in championship competitions during the outdoor season, which lasts for about five months.

TEST SHOOTING

In the test shooting bout each participant shot 25 shots on each of two different days within one week just before and after the intervention (that is, a total of 2500 shots). All the test shootings were done at a local club under standardised conditions, standing shooting at $15 \mathrm{~m}$ distance at the same indoor range with identical rifle and clothing. The shooting conditions were approved and attested to by an authorised person from the shooting club. The targets were sent to NRAN to be assessed independently. The sums of the vertical and horizontal dispersion of the shots, as read through the centre of the bullet hole, were calculated and given as height plus width (in $\mathrm{mm})$.

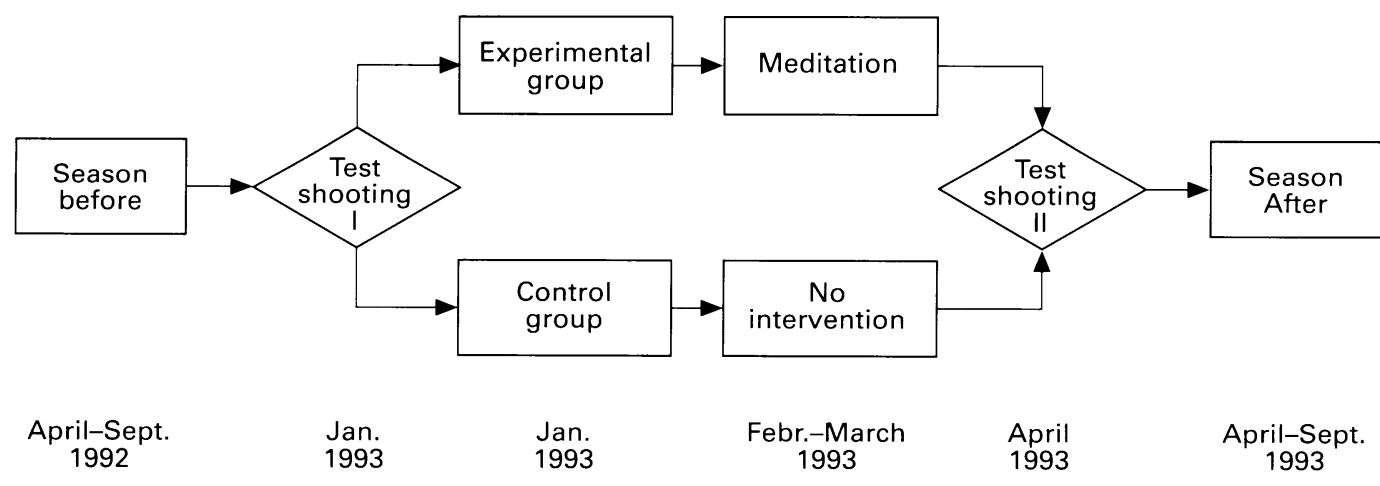

Figure 3 Flow chart showing time sequence of the study. 
TEST OF TENSION

The relation between tension and performance was studied in all the test shootings. Just after each test, the participants evaluated their own tension on a visual analogue scale (VAS) from 1-7 ( 1 is "very low tension" and 7 is "very high tension"). Although there is little empirical evidence for using such a "one off" assessment of tension, there are indications ${ }^{16}$ that a self recorded experience of tension may be clinically as relevant as objective measures like EMG. All the participants were taught how to fill out the scheme at an introductory meeting before the first test shooting bout. In total 100 schemes were finally evaluated.

\section{MANIPULATION CHECK}

The experimental group completed a questionnaire ${ }^{17}$ just after the intervention, reporting compliance to regular practice of meditation. Five alternatives (practised $>41$ times per month; 31-40 times per month; 21-30 times per month; 11-20 times per month and $<10$ times per month) were presented in the scheme. They were also asked if they had experienced specific signs of relaxation during meditation ("..muscles is getting heavy and warm, an increased amount of tear fluids or falling asleep"). The latter was done as an additional compliance control. The reasons for questioning about "signs of relaxation" were deliberately not explained to the participants, so as not to induce them to answer yes.

\section{HABITUATION}

The shooting results from the four tests were compared to exclude a possible habituation to the test situation.

POSSIBLE CONFOUNDING VARIABLES

Any factor influencing level of performance, tension, and effects of relaxation may be seen as a possible confounding variable in this study.

Smoking was considered because it is well known that smoking is a sympathetic stimulating agent. When discussing effects of relaxation on a tension sensitive performance, it might be that a change in smoking habits could affect the outcome. Smoking also affects the $\mathrm{O}_{2}$ tension and $\mathrm{VO}_{2} \mathrm{max}$, thus affecting aerobic sports at least.

Registration of life event stress ${ }^{18}$ included questions about "illness", which affects performance. We also inquired about stress in general, as having a possible influence on performance, for example through depression of the immune system. ${ }^{1920}$

An evaluation of general health was included, since wellbeing might be influenced by meditation and may itself influence the level of performance.

Life event stress was recorded by a shortened 60 question version ${ }^{18}$ of a life event stress questionnaire $^{21}$, a test of general health, ${ }^{22}$ and a questionnaire on smoking habits.

PRESSURE TO PERFORM

Hawthorne's effect ${ }^{23}$ might be expected with the increased attention given to the experimen- tal group, perhaps leading to an increased expectancy to perform. Therefore, all the participants in this group were requested to fill in a questionnaire regarding their feeling of extra pressure to perform well because they were in the experimental group. A scale from $1-7$, where 1 is "not at all" and 7 is "to a very high degree", was used for this purpose.

This study was deliberately not placebo controlled. Such a control would have been desirable for research purposes. However, it is questionable whether it is possible to use a placebo group to control a meditation group intervention at all. Under the circumstances, the design chosen might be seen as being closer to reality. The study is measuring a real situation: learning a meditation technique versus not learning such a technique. Evidently, the social and pedagogic setting in framing the meditation course also plays a role in the outcome.

STATISTICS

Parametric (ANOva and Student's $t$ test) and non-parametric (Wilcoxon rank sum test) statistics were used to determine the significance of observed differences between the two groups. Bartlett's test for homogeneity of variance was used to determine which type of test to use in the presentation. Pearson's coefficient of correlation was performed to determine the degree and significance of the linear relationship between the variables "tension" and "performance".

\section{Results}

\section{SHOOTING RESULTS}

The competition score the season after (1993) was compared with the score the season before the intervention (1992). The differences in scores from 1992 to 1993 were significantly better in the meditation group [+ 0.62 (SEM $0.6)$ points] than in the control group $[-1.5$ (0.8) points) $(\mathrm{P}<0.05)$ (fig 1$).$

No significant difference between the meditation group [+5.2 (7.9) $\mathrm{mm}]$ and the control group [-7.8 (11.4) $\mathrm{mm}$ ] was observed in the test shootings before and after the intervention (fig 2).

\section{RECORDS OF TENSION}

There was a positive correlation between low tension during the test shootings and a good performance, displayed in fig 4 as a quadratic regression (calculated as a regression line, $r=$ $0.42, \mathrm{P}<0.0001)$. A significant difference regarding the performance emerges between tension category 2 compared to category 3-6 ( $\mathrm{z}$ $=-3.36, \mathrm{P}<0.001$, as calculated by the Wilcoxon rank sum test).

COMPLIANCE AND PRESSURE TO PERFORM

The experimental group had practised meditation 36 times per month on average during the intervention, and 11 of $13(85 \%)$ had experienced signs of relaxation after meditation. They answered mean 3.4 on the scale of whether they felt extra pressure to do well because they were in the experimental group. 


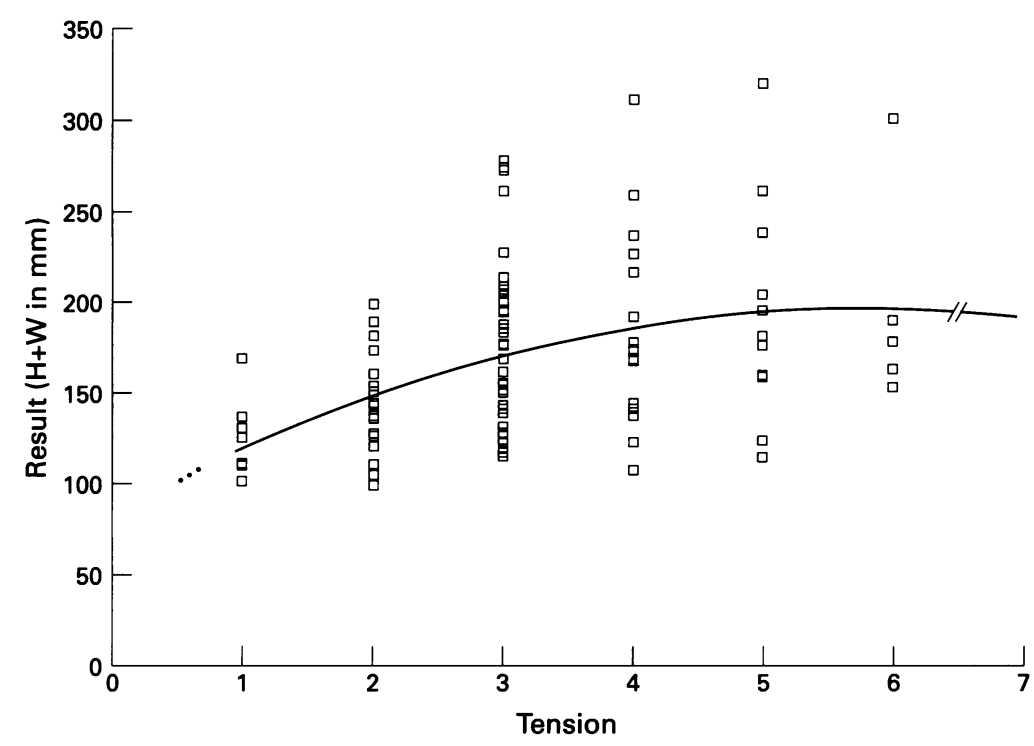

Figure 4 The shooting results in all the tests $v$ self reported tension on a VAS (based on 100 registrations, 25 participants shooting four times each).

\section{HABITUATION}

The test results of both groups taken together were about the same from test day 1 to test day $4(r=-0.01, \mathrm{P}=0.90)$.

\section{CONFOUNDING VARIABLES}

The number of smokers and the stress burden were very small. No significant differences between the groups were found (table). No change was seen in the general health questionnaire either between groups or in the intragroup analysis.

\section{Discussion}

The main result in this study was that, in comparison to the control group, the meditation group significantly improved their competition shooting the season after the intervention (1993) $v$ the season before (1992). There was no significant improvement in the test shootings when comparing the groups. The results merge after the intervention, probably because of regression to the mean.

For a majority of the shooters it is reasonable to assume that the pressure on the performer gets harder the more important the competition is, although this will be related to experience, level of competition, and motivation. An increased stress load as experienced in competitions might accelerate the need for a stress reducing technique. ${ }^{24}$

A positive association between a self experience of low tension and a good performance in the test shooting bouts was shown Whether the shooters shot well because they had a low level of tension, or became relaxed

Possible confounding variables, mean (SEM)

\begin{tabular}{llllll}
\hline & \multicolumn{2}{l}{ Control group $(n=12)$} & & \multicolumn{2}{l}{ Meditation group $(n=13)$} \\
\cline { 2 - 3 } & Before & After & & Before & After \\
\hline Smoker n & 2 & 2 & & 2 & 2 \\
General health & $53.2(1.7)$ & $51.5(1.5)$ & & $52.5(2.6)$ & $50.7(2.9) \mathrm{NS}$ \\
Stress $^{\star \star}$ & $2.1(1.0)$ & $0.8(0.7)$ & & $2.0(1.5)$ & $1.9(1.1) \mathrm{NS}$ \\
\hline
\end{tabular}

^ Tested by General Health Questionnaire (GHQ-28).

$\star \star$ Test by Life Event Stress Test (sumscore). because they shot well, is uncertain. They were not aware of their own results, however, when they assessed their level of tension during the shooting. The knowledge of a good result could not be responsible for low tension unless they just felt that they shot well. The first explanation thus seems more likely. The significant relation between good performance and low tension supports theories of a peak or ideal state of performance: The performance is at its best when the muscles are relaxed and loose and the performer feels calm and quiet. ${ }^{25} 26$

Eighteen per cent $\left(=r^{2}\right)$ of the variance in performance was explained by the level of tension in the present data. In elite sports, this is a considerable figure which underlines the importance of coping with tension to achieve an optimal level.

Our results should be interpreted with caution. Candidates were selected through invitations to five shooting clubs only. There is no reason to believe, however, that this would bias the results. Secondly, probably only shooters motivated for this kind of programme took part in the study. The study was, however, well controlled and had no dropouts. No subjects were excluded because they had too little practice in the technique. While the study is small, compliance was very high. Compliance regarding the practice of the relaxation techniques was satisfactory. Nearly all participants in the meditation group experienced signs of relaxation, an additional indicator of a satisfactory practice. The impression of high compliance was supported by the instructors in meditation. Habituation to the test situation did not seem to be a confounding factor. The groups shot similar scores from test day 1 to test day 4 , providing evidence of high reliability. The data did not suggest that the participants in the experimental group felt extra pressure to act well.

Since elite shooters were studied, only a very small margin of improvement was expected. The results of meditation were probably also minimised because the intervention was not given in collaboration with the shooting trainer, and the participants did not have the chance to choose which group to attend. Research indicates that a multifactorial intervention will be of benefit to the athlete. ${ }^{1}$ It is possible that a cognitive intervention added to the meditation technique might have enhanced the outcome. The possible confounders were very small, evenly distributed, and probably did not influence the results in the study. The two groups of shooters did not engaged themselves unevenly in other kinds of mental training that could have affected the results. The results from our study support recent reviews claiming that mental training has a beneficial effect in sports. ${ }^{1-3}$

\section{CONCLUSION}

Meditation practice may enhance the competition performance in shooters. An association between a self experience of tension and a good performance was observed. The performance was, to a considerable degree, associated with the level of tension. 
We thank NRAN for supporting the meditation course financially, to Odd Kjørmo for help with the references, and to Harald Arnesen and Are Holen for helpful comments.

1 Weinberg RS, Comar W. The effectiveness of psychological interventions in competitive sport. Sports Med 1994;18:406-18.

2 Vealy RS. Current status and prominent issues in sports psychology interventions. Med Sci Sports Exerc 1994.26: 495-502.

3 Greenspan MJ, Feltz DL. Psychological interventions with athletes in competitive situations: a review. Sport Psycho 1989; 3:219-28.

4 Morris T, Summers J, eds. Sports psychology. Theory, applicaMorris T, Summers J, eds. Sports psychology. Theory, appla
tions and issues. Chichester: John Wiley \& Sons, 1995.

5 Feltz DL, Landers DM. The effect of mental training on motor skill learning and performance: a meta-analysis. $\mathcal{f}$ Sports Psych 1983;5:25-57.

6 Onestak DM. The effects of progressive relaxation, mental practise and hypnosis on athletic performance: a review. $\mathcal{f}$ Sport Behav 1991;14:247-82.

7 Orlick T, Partington J. Mental links to excellence. Sports Psychol 1988;2:105- 30.

$8 \mathrm{Krenz}$ EW. Hypnosis versus autogenic training: a comparision. Am f Clin Hypnosis 1986;28:209-13.

9 Shapiro DH. Overview: clinical and physiological comparison of meditation and other self-control strategies. $A m$ Psychiatry 1982;139:267-74.

10 Murphy M, Donovan S. The physical and psychological effects of meditation. A review of contemporary meditation research of meditation. A review of contemporary meditation research
with a comprehensive bibliography 1931-1988. Bij Sur, CA: with a comprehensive biblio

11 Wallace RK. Physiological effects of transcendental meditation. Science 1970;167:1751-4.
12 Wallace $\mathrm{RK}$, Benson $\mathrm{H}$, Wilson AF. A wakeful hypometabolic state. Am f Physiol 1971;221:795-9.

13 Wallace RK, Benson H. The physiology of meditation. Sci Am 1972;226:84-90.

14 Holen A, ed. The psychology of silence (in Norwegian), 6th ed. Oslo: Dyade forlag, 1989.

15 ACEM international newsletter, vol 6, No 1. Oslo: ACEM, 1986.

16 Vasseljen $\mathrm{O}$. Work-related shoulder and neck pain with reference to muscle activity, individual factors, and psychosocial exposures. Trondheim: The University of Trondheim, Norway, 1995. Doctoral dissertation.

17 Solberg EE, Halvorsen R, Sundgot-Borgen J, Ingjer F, Holen A. Meditation: a modulator of the immune response to physical stress? Br $\mathcal{F}$ Sports Med 1995;29:255-7

18 Holen A. A long-term outcome study of survivors from a disasHolen A. A long-term outcome study of survivors from a disas-
ter. Oslo: University of Oslo, 1990. Doctoral dissertation.

19 Ader R, Cohen N, Felten D. Psychoneuro-immunology: interactions between the nervous system and the immune system. Lancet 1995;345:99-103.

20 Nieman DC. Exercise, infection and immunity. Int $\mathcal{f}$ Sports Med 1994;15:131- 41.

21 Holmes TH, Rahe RH. The social readjustment scale. $\mathcal{f}$ Psychosom Res 1967;11:213-8

22 Goldberg D, Williams P. A user's guide to the General Health Questionnaire. NFER-Nelson, 1988.

23 Mayo E. The social problem of an industrial civilisation. London: Routledge, 1949.

24 Lazarus RS, Folkmann S. Stress, appraisal and coping. New York: Springer, 1984.

25 Hanin YL. Interpersonal and intragroup anxiety in sports. In: Hackfort D, Spielberger C, eds. Anxiety in sports: an interpersonal perspective. New York: Hemisphere, 1989:1925.

26 Loehr JE. Mental toughness training for sports: achieving athletic excellence. Lexington: Greene, 1986. 